\title{
Oesophageal carcinoma: a neglected volcano in Sri Lanka
}

\author{
Ishanka Ayeshwari Talagala \\ Ministry of Health, Nutrition and Indigenous Medicine, Sri Lanka \\ Correspondence: drishanka@gmail.com
}

DOI: https://doi.org/10.4038/jccpsl.v24i2.8166

Received on: 03 July 2018

Accepted on: 26 September 2018

"Ms X, a 37-year-old teacher and mother of two young children (three years old and five months old) found out that her nausea and vomiting were not resolved even following the delivery of her second child. In addition, she was suffering from difficulty in swallowing and generalized weakness as well, for which she was re-assured by her family, attributing these features to her lactation and the possibility of another pregnancy. With a 'negative' pregnancy test, she sought medical care and underwent an upper gastro-intestinal endoscopy test, which revealed a mass in the middle one third of the oesophagus. Histology confirmed this mass to be oesophageal carcinoma. She lost her life eight months following this diagnosis".

High morbidity, mortality and financial burden due to cancer have clutched the attention of policy makers throughout the globe, particularly in low- and middleincome countries, which account for $77 \%$ of the global disability-adjusted life years (DALYs); $78 \%$ of years of life lost (YLLs); and $71 \%$ of the cancer deaths (1-3).

In Sri Lanka, cancer poses a great impact on the economy of individuals, households as well as the country, with the care being predominantly provided by the state and increasingly being financed out-ofpocket by family (4). Oral cavity, breast, cervix and oesophageal cancer are reported to be the commonest cancer types in Sri Lanka (5), of which the latter continues to be the third commonest cancer among males and fifth commonest among females (6). It is shown that the incidence of oesophageal carcinoma in Sri Lanka has been gradually, yet steadily increasing in the recent past. This increase is most likely to be due to a change in the histological trend from squamous cell carcinoma to adenocarcinoma which follows a more aggressive disease progression, further fuelling the burden due to oesophageal carcinoma $(5,7)$. It is important to note that oesophageal carcinoma lacks a specific prevention programme, unlike in the case of oral, breast and cervical cancers. Considering these facts, oesophageal carcinoma is presumably a volcano that has been neglected in the country, and awaits its eruption to cause severe damage. This catastrophe can be prevented by prompt action taken through primary, secondary and tertiary prevention modalities against oesophageal carcinoma in Sri Lanka.

\section{Primary prevention: identifying population- specific risk factors}

The underlying causes of oesophageal carcinoma are multi-factorial. As with any other chronic noncommunicable disease, the conventional risk factors are related to non-modifiable demographic (e.g. older age, male sex, genetic predisposition) (8-9) and socioeconomic status (e.g. low socio-economic status) (1011); clinical conditions such as gastro-oesophageal reflux disease (12) and Barrett's oesophagus (13); medication such as non-steroidal anti-inflammatory drugs (NSAIDs) and aspirin (14); and lifestyle factors such as physical inactivity (15), tobacco, alcohol, and low intake of fruits and vegetables (16-17). In addition to these, several other risk factors have been newly 
identified in the recent past, which are related to specific elements in the diet (e.g. diet deficient in vitamins A, B, C and E, selenium and beta carotene) (18-19); food preparation methods (e.g. chillies and spicy food) (18, 20); consumption of food/drinks at high temperatures (21); drinking water sources (22); chewing betel leaf with tobacco (23) and arecanut (24); and exposure to ionizing radiation (25). However, evidence on these risk factors is largely from the developed countries and may not be directly applicable to the risk profile in developing countries including Sri Lanka. Furthermore, there may be other risk factors unique to developing countries, owing to practices related to agriculturebased settings and occupations (e.g. indiscriminate use of agrochemicals and other occupational hazards).

Currently, there is minimal research evidence on the risk factors of oesophageal carcinoma in Sri Lanka, which could assist health care planners to provide targeted primary prevention for oesophageal carcinoma. In bridging this knowledge gap, a hospital-based unmatched case-control study was conducted among 49 incident cases of oesophageal carcinoma and 196 ambulatory controls powered enough to detect the smallest reported risk for oesophageal carcinoma (odds ratio of 2.5 for consumption of alcohol) (26). The cases were newly diagnosed oesophageal carcinoma patients recruited from the National Cancer Institute Maharagama. Controls were those without oesophageal carcinoma confirmed by negative findings of upper gastro-intestinal endoscopy (UGIE) examination (apparently healthy oesophageal mucosa, gastrooesophageal junction and the stomach up to the distal duodenal sphincter with no macroscopic changes, erosions or lesions) (27). They were recruited from the Endoscopy Unit of the National Hospital of Sri Lanka. Several exclusion criteria were considered to avoid early disease, pre-malignant stages and shared risk factors of other cancers. In both cases and controls, several potential risk factors were assessed using valid and reliable tools.

After controlling for confounders and interactions using logistic regression (LR) analysis, populationspecific risk profile for oesophageal carcinoma was identified. This risk profile included: age more than 65 years; family history of cancer; sub-optimal consumption of fibre in the diet; sub-optimal consumption of antioxidants in the diet; over-consumption of deep fried food; 'low' total lifetime sports and exercise activities; 'high risk' alcohol consumption; ever betel quid chewing; exposure to agrochemicals; consumption of pipe-borne water as the major source of water; and ever exposure to radiation (Table 1). Most of these risk factors are highly prevalent among Sri Lankan populations and therefore, it is essential that public health professionals pay more attention to empower the general public on lifestyle and environmental modifications relevant to oesophageal carcinoma.

Table 1. Risk factor profile of oesophageal carcinoma

\begin{tabular}{lccc}
\hline & & & $\mathbf{9 5 \%}$ CI \\
\cline { 2 - 4 } Independent variable & Odds & Lower & Upper \\
\hline Age more than 65 years & 3.99 & 1.12 & 14.25 \\
Family history of cancer & 5.04 & 1.34 & 19.00 \\
Sub-optimal consumption of fibre & 3.58 & 1.10 & 12.32 \\
Sub-optimal consumption of antioxidants & 7.01 & 2.18 & 22.54 \\
Sub-optimal consumption of deep fried food & 6.68 & 1.98 & 22.59 \\
'Low' level of total lifetime sports and exercise activities & 5.83 & 1.48 & 23.03 \\
'High risk' alcohol consumption & 11.71 & 2.78 & 49.43 \\
Ever betel quid chewing & 6.11 & 1.90 & 20.00 \\
Exposure (direct/indirect) to agrochemicals & 6.57 & 1.42 & 30.30 \\
Pipe-borne water as the major drinking source & 5.62 & 1.67 & 18.88 \\
Ever exposure to radiation & 4.64 & 1.38 & 15.53 \\
\end{tabular}




\section{Secondary prevention: risk prediction tool for early diagnosis}

As with other cancers, the prognosis of oesophageal carcinoma depends largely on its stage of disease (28). It is shown that the five-year survival rates for stages I, II and III are 50-80\%; 30-40\%; and $10-15 \%$, while stage IV has a median survival of less than one year (11). Since it is a fast growing tumour with a potentially high cell doubling time, even a delay of a few months in diagnosis would affect the prognosis substantially (29). As such, every effort should be taken to diagnose the disease as early as possible, so that the outcome could be considerably better than when detected late. However, clinical symptoms of oesophageal carcinoma are well-known to appear late. Substantial circumferential involvement and considerable penetration into the oesophageal lumen act as pre-requisites for dysphagia and the tumour spreading to adjacent structures to present with pain (30).

Limiting the number of individuals in advanced disease stages is achieved by means of early diagnosis and early initiation of relevant treatment. This could be accomplished by a well-organized screening program for oesophageal carcinoma, which is a secondary prevention strategy to address the issue of late diagnosis. The recommended screening is routine UGIE followed by histological confirmation (31). However, this is routinely practiced in the world only in inherently highrisk areas of China, owing to its high cost and the limited availability of trained human resources and equipment. For example, in Sri Lanka, endoscopy units are available in only 61 hospitals with only 12 consultant gastroenterologists and 144 consultant surgeons providing services on UGIE examination (S Wimalarathne, personal communication, 17 June 2016). In addition, invasiveness of the UGIE examination plays a crucial role in poor compliance of individuals to undergo screening. As a solution, a simple, low-cost, non-invasive, easily administrable screening tool (i.e. a risk prediction model) could be introduced as a primary screening strategy to identify the most at-risk persons for oesophageal carcinoma among the general population, so that only those identified could be subsequently offered UGIE as a secondary screening strategy for endoscopic confirmatory diagnosis, thus, preventing the delays in diagnosis of the disease. Such screening tools will address the existing gaps in secondary prevention of oesophageal carcinoma especially in low-resource settings.
Risk prediction models that are already in use in the world for screening for oesophageal carcinoma, have been developed based on non-modifiable and modifiable risk factors specific for geographical regions, and include a variety of predictors in relation to genetic variants (25 single nucleotide polymorphisms), socio-economic factors (age, sex, level of education), lifestyles (smoking and alcohol), anthropometry, and clinical features (gastro-oesophageal reflux, use of acid suppressant medications and NSAID) (32-33). As these models have their own restrictions to be used in other settings, it is mandatory that countries develop their own population-specific risk prediction tools to identify those at risk of developing oesophageal carcinoma.

In an attempt to develop a simple risk prediction tool to identify those at increased risk of oesophageal carcinoma, a hospital-based unmatched case-control study was conducted among Sri Lankans, utilizing the newly developed population-specific risk factor profile. For this purpose, 30 cases and 138 controls were recruited to achieve $98 \%$ expected sensitivity and $90 \%$ expected specificity. Recruitment and sampling of subjects was similar to the previously described case-control study (27). When developing the risk prediction model, all the risk factors significant in the previous study as well as those not significant but clinically important according to previous studies were considered.

The best model that was derived using receiver operating characteristic (ROC) curve, described 0.97 (95\% CI=0.94-0.99) area under the curve. This risk prediction model is shown in Table 2. The risk score for identifying those at risk of oesophageal carcinoma from those without was 34.5 at $96.7 \%$ sensitivity and $84.1 \%$ specificity, indicating good predictive ability of the risk prediction tool. This risk prediction model was further tested for judgmental validity by a panel of experts using modified Delphi technique. It demonstrated high reliability (Spearman correlation coefficient $=0.81$ ) as well during split-half cross-validation.

\section{Tertiary prevention: improving the quality of life}

The primary focus of any cancer treatment is to improve the quality of life $(\mathrm{QoL})$ either by curing the disease or by ameliorating the worst symptoms as long as possible. Improving the QoL in oncology patients 
Table 2. Risk prediction model for assessing the risk of oesophageal cancer

\begin{tabular}{llc}
\hline Predictor variable & Categories & Weighted score $^{1}$ \\
\hline Age of the individual & $>65$ years & $4^{2}$ \\
Lifetime sports and exercise activities & Low level & $6^{2}$ \\
Alcohol consumption & High risk & $12^{2}$ \\
Consumption of dietary Fibre & Sub-optimal & $4^{2}$ \\
Consumption of antioxidants & Sub-optimal & $7^{2}$ \\
Consumption of deep fried food & Over-consumption & $7^{2}$ \\
Family history of cancer & Yes & $5^{2}$ \\
Ever betel quid chewing & Yes & $6^{2}$ \\
Ever exposure to radiation & Yes & $5^{2}$ \\
Exposure (direct/indirect) to agrochemicals & Yes & $7^{2}$ \\
Drinking water source & Pipe-borne & $6^{2}$ \\
Ever tobacco smoking & Yes & $11^{3}$ \\
\hline Total score & & $\mathbf{8 0}$ \\
\hline
\end{tabular}

1 Weighted score based on the odds ratio obtained for each variable

2 Based on the adjusted odds ratio of the population-specific risk factor profile for oesophageal carcinoma

3 Based on the odds ratio of the bivariate analysis

has become an important therapeutic goal, with most treatment decisions being heavily influenced by their effect on patients' QoL (34). Oesophageal carcinoma is well-known for poor prognosis, with its five-year survival rate being as low as $5-10 \%$ (35). However, the advancements in treatment modalities in the last few decades have drastically improved the survival of these patients (36), but how this improvement has affected their QoL remains inconclusive (37).

The national policy for cancer prevention and control in Sri Lanka aims at a comprehensive programme (38), which includes the provision of palliative care services for cancer survivors through universal health coverage. Though many initiatives have been undertaken, further improvement of these services has been hampered due to the lack of evidence on the improvement in QoL following treatment which would otherwise enable targeted palliative care services as a tertiary preventive strategy. To provide this muchneeded evidence, a prospective cohort study was conducted at the National Cancer Institute Maharagama among 51 newly diagnosed patients of oesophageal carcinoma who had not yet undergone the initial treatment (surgery, chemotherapy and/or radiotherapy). The majority were males $(70.6 \%)$, over 60 years of age (53\%), employed (60.8\%) and of low social status (social classes IV and V) (64.7\%). Their QoL was assessed and compared before (baseline) and one month after the completion of initial treatment (follow-up irrespective of the treatment plan), using three questionnaires validated for Sri Lanka (EORTC QLQ-C30 core-questionnaire, EORTC QLQ-OES18 modules specific for oesophageal carcinoma and questionnaire on family support (39-41). One month was considered long enough to avoid the temporary effect of side effects during treatment phase on their QoL. The QoL dimensions that showed a significant change (improvement or deterioration) following the initial treatment are given in Table 3. Worsening aspects in the QoL following initial treatment were physical, emotional, social and role functioning as well as the general symptoms, financial difficulties and reduction of family support. Dysphagia seemed to improve significantly following the initial treatment in contrast to dry mouth. 
Table 3. The dimensions in QoL that showed a significant change after initial treatment

\begin{tabular}{|c|c|c|c|c|c|c|}
\hline \multirow[t]{2}{*}{ Scale } & \multicolumn{2}{|c|}{ Baseline score } & \multicolumn{2}{|c|}{ Follow up score } & \multirow[b]{2}{*}{$\begin{array}{c}\text { Mean } \\
\text { difference }^{4}\end{array}$} & \multirow[b]{2}{*}{$95 \% \mathrm{CI}$} \\
\hline & $\begin{array}{l}\text { Mean } \\
(\mathrm{N}=51)\end{array}$ & SD & $\begin{array}{c}\text { Mean } \\
(\mathrm{N}=\mathbf{4 1})^{3}\end{array}$ & SD & & \\
\hline Physical functioning ${ }^{1}$ & 53.1 & 28.8 & 34.3 & 24.5 & -20.8 & $-30.0,-11.6$ \\
\hline Role functioning ${ }^{1}$ & 42.2 & 34.5 & 21.5 & 26.2 & -22.8 & $-34.7,-10.8$ \\
\hline Emotional functioning ${ }^{1}$ & 53.4 & 26.4 & 42.1 & 31.2 & -14.0 & $-24.4,-3.7$ \\
\hline Social functioning ${ }^{1}$ & 57.2 & 23.2 & 37.4 & 26.8 & -23.2 & $-31.9,-14.4$ \\
\hline Family support ${ }^{1}$ & 82.6 & 20.4 & 80.5 & 14.4 & -6.5 & $-10.6,-2.44$ \\
\hline Fatigue $^{2}$ & 52.1 & 28.9 & 71.3 & 28.8 & 20.9 & $9.8,31.9$ \\
\hline Dysphagia $^{2}$ & 54.0 & 27.0 & 37.1 & 29.0 & -13.6 & $-22.9,-4.2$ \\
\hline Dry mouth ${ }^{2}$ & 39.2 & 34.5 & 58.5 & 37.1 & 20.3 & $7.9,32.7$ \\
\hline Nausea \& vomiting ${ }^{2}$ & 40.2 & 34.4 & 54.1 & 37.6 & 13.0 & $0.7,25.3$ \\
\hline Pain $^{2}$ & 45.4 & 32.7 & 71.5 & 28.7 & 27.6 & $15.8,39.5$ \\
\hline Insomnia ${ }^{2}$ & 33.3 & 32.0 & 43.9 & 35.3 & 13.8 & $2.3,25.3$ \\
\hline Loss of appetite ${ }^{2}$ & 42.5 & 34.0 & 54.5 & 37.8 & 13.0 & $0.4,25.7$ \\
\hline Constipation $^{2}$ & 30.1 & 35.4 & 47.9 & 41.5 & 16.3 & $5.5,27.1$ \\
\hline Financial difficulties ${ }^{2}$ & 50.9 & 31.5 & 77.2 & 21.6 & 30.9 & $20.5,41.3$ \\
\hline
\end{tabular}

${ }^{1}$ Scores range from 0-100, with higher scores indicating a higher level of functioning/family support

${ }^{2}$ Scores range from 0-100, with higher scores indicating a higher degree of symptoms/financial difficulties

${ }^{3}$ Ten patients died during the follow up period

${ }^{4}$ A negative mean difference indicates deterioration of functioning, family support and improvement of symptoms while a positive mean difference indicates worsening of symptoms and financial difficulties

\section{Evidence for improved health services}

It is justifiable to consider oesophageal carcinoma as a neglected volcano in Sri Lanka, based on the evidence derived on the risk factors and quality of life of patients with oesophageal carcinoma.

The national policies and strategic frameworks on non-communicable diseases and cancer prevention and control identify the importance of targeted primary preventive measures to prevent cancer as well as early detection at primary healthcare level through screening of asymptomatic populations and prompt referral of the suspicious ones for confirmation of diagnosis and further management $(38,42)$. In line, knowledge on the conventional as well as newly identified risk factors for oesophageal carcinoma enables the health policy makers to prioritize evidence-based primary prevention activities in the country, and thereby utilize the limited resources to a maximum to reduce the future burden of this disease. Also, the burden caused by advanced disease condition can be controlled by adopting the simple low cost risk prediction tool that would enable the identification of those at risk of developing oesophageal carcinoma, who could then be referred for the UGIE assessment.

The national policy on cancer further elaborates on providing disease-specific and patient-centred palliative care. Evidence on different aspects of QoL of patients with oesophageal carcinoma that may change over time should shape the management aspects of these patients towards tertiary prevention strategies. 


\section{References}

1. Institute of Medicine (US) Committee. In Sloan FA \& Gelband H (Eds.), Committee on Cancer Control Opportunities in Low- and Middle-Income Countries. Washington (DC): National Academies Press, 2007.

2. WHO. Fact Sheets Cancer. World Health Organization, 2018.

Available from: http://www.who.int/news-room/factsheets/detail/cancer on 03/06/2018

3. Bloom DE, Cafiero ET, Jané-Llopis E, AbrahamsGessel S, Bloom LR, Fathima S, Weinstein C. The Global Economic Burden of Non-communicable Diseases. Geneva: World Economic Forum and Harvard School of Public Health, 2011.

4. World Bank. Tackling non-communicable diseases in Sri Lanka. 2011.

Available from: http://www.worldbank.org/en/news/ feature/2012/05/16/non-communicable-diseases-srilanka

5. NCCP. Cancer Incidence Data Sri Lanka 2010. Colombo: National Cancer Control Programme, Ministry of Health, 2016.

6. NCCP. Cancer Incidence Data Sri Lanka 2009. Colombo: National Cancer Control Programme, Ministry of Health, 2015.

7. NCCP. Cancer Incidence Data Sri Lanka 2008. Colombo: National Cancer Control Programme, Ministry of Health, 2014.

8. Akbari MR, Malekzadeh R, Nasrollahzadeh D, Amanian D, Sun P, Islami F, Narod SA. Familial risks of esophageal cancer among the Turkmen population of the Caspian Littoral of Iran. International Journal of Cancer 2006; 119(5): 1047-1051.

9. GLOBOCAN. Estimated cancer incidence, mortality and prevalence worldwide in 2012. IARC Cancer Base No. 11, Fact sheets, 2012.

Available from: http://globocan.iarc.fr

10. Brown LM, Hoover R, Silverman D, Baris D, Hayes R, Swanson GM, Fraumeni Jr JF. Excess incidence of squamous cell esophageal cancer among US Black men: role of social class and other risk factors. American Journal of Epidemiology 2001; 153(2): 114-122.

11. Subasinghe D \& Samarasekera DN. Delay in the diagnosis of esophageal carcinoma: experience of a single unit from a developing country. Indian Journal of Cancer 2010; 47(2): 151-155.

12. Solaymani-Dodaran M, Logan RF, West J, Card T, Coupland C. Risk of oesophageal cancer in Barrett's oesophagus and gastro-oesophageal reflux. Gut 2004; 53(8): 1070-1074.
13. Shaheen N \& Ransohoff DF. Gastroesophageal reflux, Barrett esophagus, and esophageal cancer: scientific review. Journal of American Medical Association 2002; 287(15): 1972-1981.

14. Corley DA, Kerlikowske K, Verma R, Buffler P. Protective association of aspirin/NSAIDs and esophageal cancer: a systematic review and metaanalysis. Gastroenterology 2003; 124(1): 47-56.

15. Singh S, Devanna S, Edakkanambeth Varayil J, Murad MH, Iyer PG. Physical activity is associated with reduced risk of esophageal cancer, particularly esophageal adenocarcinoma: a systematic review and meta-analysis. BMC Gastroenterology 2014; 14: 101.

16. Cook-Mozaffari PJ, Azordegan F, Day NE, Ressicaud A, Sabai C, Aramesh B. Oesophageal cancer studies in the Caspian Littoral of Iran: results of a case-control study. British Journal of Cancer 1978; 39(3): 293-309.

17. Prabhu A, Obi KO, Rubenstein JH. The synergistic effects of alcohol and tobacco consumption on the risk of esophageal squamous cell carcinoma: a metaanalysis. The American Journal of Gastroenterology 2014; 109(6): 822-827.

18. Kamangar F, Chow W-H, Abnet C, Dawsey S. Environmental causes of esophageal cancer. Gastroenterology Clinics of North America 2009; 38(1): 27-57.

19. Tran GD, Sun XD, Abnet CC, Fan JH, Dawsey SM, Dong ZW, Taylor PR. Prospective study of risk factors for esophageal and gastric cancers in the Linxian general population trial cohort in China. International Journal of Cancer 2005; 113(3): 456463.

20. Phukan RK, Chetia CK, Ali MS, Mahanta J. Role of dietary habits in the development of esophageal cancer in Assam, the North-Eastern Region of India. Nutrition and Cancer 2001; 39(2): 204-209.

21. Islami F, Boffetta P, Ren JS, Pedoeim L, Khatib D, Kamangar F. High-temperature beverages and foods and esophageal cancer risk: a systematic review. International Journal of Cancer 2009; 125(3): 491-524.

22. Xibib S, Meilan H, Moller H, Evans HS, Dixin D, Wenjie D, Jianbang L. Risk factors for oesophageal cancer in Linzhou, China: a case control study. Asian Pacific Journal of Cancer Prevention 2003; 4(2): 119-124.

23. Nayar D, Kapil U, Joshi YK, Sundaram KR, Srivastava SP, Shukla NK, Tandon RK. Nutritional risk factors in esophageal cancer. The Journal of the Association of Physicians of India 2000; 48(8); 781-787.

24. Akhtar, S. Areca nut chewing and esophageal squamous-cell carcinoma risk in Asians: a metaanalysis of case-control studies. Cancer Causes Control 2013; 24(2): 257-265. 
25. Wang J-X, Inskip PD, Boic Jr JD, Li B-X, Zhang J-Y, Fraumeni Jr JF. Cancer incidence among medical diagnostic X-ray workers in China, 1950 to 1985. International Journal of Cancer 1990; 45(5): 889-895.

26. Yang CX, Wang HY, Wang ZM, Du HZ, Tao DM, Mu XY, Tajima K. Risk factors for oesophageal cancer: a case control study in South-Western China. Journal of Cancer Prevention 2005; 6(1): 48-53.

27. Talagala IA. Risk factors, risk prediction and quality of life of oesophageal carcinoma among adults of the Western Province. MD thesis (Community Medicine). Colombo: Post Graduate Institute of Medicine, Sri Lanka, 2016.

28. American Joint Committee on Cancer. AJCC Cancer Staging Manual (6 $6^{\text {th }}$ ed.), USA, 2002.

29. Haustermans K, Fowler F, Geboes K, Lerut T, van der Schueren E. Do cell kinetics have prognostic and/or predictive value in oesophageal cancer treated by surgery? European Journal of Surgical Oncology 1997; 23(4): 293-297.

30. Bird-Lieberman EL \& Fitzgerald RC. Early diagnosis of oesophageal cancer. British Journal of Cancer 2009; 101: 1-6.

31. Zheng X, Mao X, Xu K, Lü L, Peng X, Wang M, Xu G, Hua Z, Wang J, Xue H. et al. Massive endoscopic screening for esophageal and gastric cancers in a high-risk area of China. PLOS ONE 2015; 10(12): e0145097.

32. Esophageal cancer prevention. Available from: http://www.cancer.gov/cancertopics/ pdq/prevention/esophageal/HealthProfessional)

33. Chang J, Huang Y, Lixuan Wei L, Baoshan Ma B, Miao X, Li Y, Hu Z, Yu D, Jia W, Liu Y. et al. Risk prediction of esophageal squamous-cell carcinoma with common genetic variants and lifestyle factors in Chinese population. Carcinogenesis 2013; 34(8): 1782-1786.

34. Soni MK \& Cella D. Quality of life and symptom measures in oncology: an overview. The American Journal of Managed Care 2002; 8(18 Suppl): S560573.
35. Marmot M, Atinmo T, Byers T, Chen J, Hirohata T, Jackson A, James W, Kolonel L, Kumanyika S, Leitzmann C. et al. Food, Nutrition, Physical Activity and the Prevention of Cancer: a Global Perspective (WCRF/AICR Expert Report). Washington DC: World Cancer Research Fund/American Institute for Cancer Research, 2007.

36. Law S, Kwong DLM, Kwok K-F, Wong K-H, Chu KM, Sham JST, Wong J. Improvement in treatment results and long-term survival of patients with esophageal cancer: impact of chemo-radiation and change in treatment strategy. Annals of Surgery 2003; 238(3): 339-348.

37. Yajuan LV, Zhang J, Qiao L. Quality of life in patients with esophageal cancer receiving definitive chemoradiotherapy or esophagectomy. Molecular and Clinical Oncology 2014; 2(5): 870-874.

38. Ministry of Health. National Policy and Strategic Framework on Cancer Prevention \& Control. Colombo: Ministry of Health, Nutrition \& Indigenous Medicine, 2015.

39. Aaronson NK, Ahmedzai S, Bergman B, Bullinger M, Cull A, Duez NJ, Filberti A, Flechtner H, Fleishman $\mathrm{SB}$, De Haes J. et al. The European Organization for Research and Treatment of Cancer QLQ-C30: a quality of life instrument for use in international clinical trials in oncology. Journal of the National Cancer Institute 1993; 85(5): 365-376.

40. Blazeby JM, Williams MH, Brookes ST, Alderson D, Farndon JR. Quality of life measurement in patients with oesophageal cancer. Gut 2006; 1995(37): 505-508.

41. Jayasekara H. An assessment of quality-of-life and satisfaction with care in patients diagnosed with some common cancers. MD Thesis (Community Medicine). Colombo: Post Graduate Institute of Medicine, Sri Lanka, 2016.

42. Ministry of Health. The National Policy \& Strategic Framework for Prevention and Control of Chronic Non-communicable Diseases. Colombo: Ministry of Health, Sri Lanka, 2009. 